\title{
A Large Retrospective Study of Epidemiological Characteristics of COVID-19 Patients in the North of Iran: Association between SARS-CoV-2 RT-PCR Ct Values with Demographic Data
}

\author{
Farzin Sadeghi $\left(\mathbb{D},{ }^{1}\right.$ Abazar Pournajaf $\mathbb{D D}^{2,3}$ Mehrdad Halaji ${ }^{1},{ }^{2,3}$ Mohammad Chehrazi, \\ Fatemeh Hejazi Amiri (D, ${ }^{3}$ Saghar Saber Amoli ${ }^{D},{ }^{3}$ Ali Hasanzadeh, ${ }^{5}$ Mostafa Javanian, \\ Masoumeh Bayani, ${ }^{2}$ Mahmoud Sadeghi Haddad Zavareh, ${ }^{2}$ Mehran Shokri, ${ }^{2}$ \\ Arefeh Babazadeh $\left(D,{ }^{2}\right.$ Mohsen Mohammadi, ${ }^{6}$ Hamed Mehdinezhad $\left(D,{ }^{7}\right.$ \\ Mahmoud Monadi, ${ }^{7}$ Parviz Amri Maleh (D), ${ }^{8}$ Hamid Reza Nouri (D), ${ }^{1}$ Abdolreza Daraei (D), \\ Mahdie Yousefnia Pasha $\left(D,{ }^{10}\right.$ Mehdi Tourani $\left(D,{ }^{11}\right.$ Seyed Raheleh Ahmadian $\left(D,{ }^{11}\right.$ \\ Nadia Esmailzadeh, ${ }^{3}$ Seyyedeh Maedeh Mirtabar, ${ }^{11}$ Shakiba Asadi, ${ }^{11}$ Ebrahim Nasiraie, ${ }^{12}$ \\ Nafiseh Ezami, ${ }^{13}$ Shahrbano Gorjinejad, ${ }^{14}$ Kobra Fallhpour, ${ }^{15}$ Fatemeh Fakhraie, ${ }^{16}$ \\ Yousef Beheshti, ${ }^{9}$ Mahnaz Baghershiroodi, ${ }^{17}$ Faeze Rasti, ${ }^{11}$ Maryam Salehi, ${ }^{3}$ \\ Atiyeh Aleahmad, ${ }^{18}$ Rahman Babapour, ${ }^{10}$ Rahim Malekzadeh, ${ }^{10}$ \\ Rahmat Habibzadeh Kashi, ${ }^{10}$ and Yousef Yahyapour $\mathbb{D}^{1}$
}

${ }^{1}$ Cellular and Molecular Biology Research Center, Health Research Institute, Babol University of Medical Sciences, Babol, Iran ${ }^{2}$ Infectious Diseases and Tropical Medicine Research Center, Health Research Institute, Babol University of Medical Sciences, Babol, Iran

${ }^{3}$ Department of Medical Microbiology, Faculty of Medicine, Babol University of Medical Sciences, Babol, Iran

${ }^{4}$ Department of Biostatistics and Epidemiology, School of Public Health, Babol University of Medical Sciences, Babol, Iran

${ }^{5}$ Department of Medical Microbiology, Faculty of Medicine, Golestan University of Medical Sciences, Gorgan, Iran

${ }^{6}$ Non-Communicable Pediatric Diseases Research Center, Health Research Institute, Babol University of Medical Sciences, Babol, Iran

${ }^{7}$ Department of Internal Medicine, Rouhani Hospital, Babol University of Medical Sciences, Babol, Iran

${ }^{8}$ Department of Anesthesiology, Rouhani Hospital, Babol University of Medical Sciences, Babol, Iran

${ }^{9}$ Department of Medical Genetics, Faculty of Medicine, Babol University of Medical Sciences, Babol, Iran

${ }^{10}$ Babol Health Center, Babol University of Medical Sciences, Babol, Iran

${ }^{11}$ Health Research Institute, Babol University of Medical Sciences, Babol, Iran

${ }^{12}$ Part of Infectious Control, Rouhani Hospital, Babol University of Medical Sciences, Babol, Iran

${ }^{13}$ Part of Medical Records, Rouhani Hospital, Babol University of Medical Sciences, Babol, Iran

${ }^{14}$ Part of Infectious Control, Amirkola Hospital, Babol University of Medical Sciences, Babol, Iran

${ }^{15}$ Part of Infectious Control, Shahid Beheshti Hospital, Babol University of Medical Sciences, Babol, Iran

${ }^{16}$ Part of Infectious Control, Shahid Yahyanejad Hospital, Babol University of Medical Sciences, Babol, Iran

${ }^{17}$ Genetics Laboratory, Shafizadeh Amirkola Children's Hospital, Babol University of Medical Sciences, Babol, Iran

${ }^{18}$ Department of Clinical Biochemistry, Faculty of Medicine, Babol University of Medical Sciences, Babol, Iran

Correspondence should be addressed to Yousef Yahyapour; uyahyapoor@yahoo.com

Farzin Sadeghi, Abazar Pournajaf, and Mehrdad Halaji contributed equally to this work.

Received 25 November 2021; Accepted 28 January 2022; Published 28 February 2022

Academic Editor: André Luiz Ferreira Costa

Copyright (c) 2022 Farzin Sadeghi et al. This is an open access article distributed under the Creative Commons Attribution License, which permits unrestricted use, distribution, and reproduction in any medium, provided the original work is properly cited. 


\begin{abstract}
Objectives. To avoid worsening from mild, moderate, and severe diseases and to reduce mortality, it is necessary to identify the subpopulation that is more vulnerable to the development of COVID-19 unfavorable consequences. This study aims to investigate the demographic information, prevalence rates of common comorbidities among negative and positive real-time reversetranscriptase polymerase chain reaction (rRT-PCR) patients, and the association between SARS-CoV-2 cycle threshold (Ct) at hospital admission, demographic data, and outcomes of the patients in a large population in Northern Iran. Methods. This large retrospective cross-sectional study was performed from 7 March to 20 December 2020. Demographic data, including gender, age, underlying diseases, clinical outcomes, and Ct values, were obtained from 8,318 cases suspected of COVID-19, who were admitted to four teaching hospitals affiliated to Babol University of Medical Sciences (MUBABOL), in the north of Iran. Results. Since 7 March 2020, the data were collected from 8,318 cases suspected of COVID-19 (48.5\% female and 51.5\% male) with a mean age of $53 \pm 25.3$ years. Among 8,318 suspected COVID-19 patients, 3,250 (39.1\%) had a positive rRT-PCR result; 1,632 (50.2\%) patients were male and $335(10.3 \%)$ patients died during their hospital stay. The distribution of positive rRT-PCR revealed that most patients $(464(75.7 \%))$ had a Ct between 21 and 30 (Group B). Conclusion. Elderly patients, lower Ct, patients having at least one comorbidity, and male cases were significantly associated with increased risk for COVID-19-related mortality. Moreover, mortality was significantly higher in patients with diabetes, kidney disease, and respiratory disease.
\end{abstract}

\section{Introduction}

The new coronavirus disease 2019 (COVID-19) pandemic was firstly observed in late December 2019 which was caused by severe acute respiratory syndrome coronavirus 2 (SARS$\mathrm{CoV}-2$ ). It was firstly found in China, rapidly spreading to other Chinese provinces and other countries [1]. In general, COVID-19 results in asymptomatic, mild, or severe respiratory tract infections in human beings. It can result in a lethal condition in some cases [2]. Up to now, various studies were conducted on COVID-19, and multiple reports from different perspectives of disease were presented, including clinical manifestations, measures for treatment, and demographics of the disease [3]. It is known that there is an association between the risk of development of severe COVID-19 and some characterized individual conditions [4].

According to a previous study, the mortality rate ranges from $1.4 \%$ to $8 \%$ in the general population, and it increases significantly in patients with specific conditions and complications. Therefore, the risk of severe symptoms, even fatal conditions, and the poorer prognosis are higher in elderly adults and individuals with comorbidities [5-8]. A large number of hospitalized people, particularly patients that are eventually hospitalized in the intensive care unit (ICU) or lose their lives, experience comorbidities such as hypertension, chronic cardiovascular disease, obesity, and diabetes [9]. If the prevalence or frequency of these underlying diseases and related comorbidities are determined, it would be useful to gain a better knowledge of the prognosis of disease, the disease treatment, and the comprehensive management of outcomes [10].

SARS-CoV-2 viral genome detection is crucially important, and real-time reverse-transcriptase polymerasechain-reaction (rRT-PCR) has served as a major and routine test to diagnose SARS-CoV-2 infection [11]. Several groups have suggested many viral targets to detect the virus, including nucleocapsid $(\mathrm{N})$, open reading frame (ORF) 1a, RNA-dependent RNA polymerase (RdRp), spike (S), and envelope (E) [12]. The severity of COVID-19 may be worsened by the virus's main load and the quantity of virus a person possesses at any one moment. Viral load is a measure of the number of viral particles present in a person [13]. A person with high SARS-CoV-2 viral loads could get worse outcomes, and according to the data from China, the viral load is higher in patients with more severe diseases $[13,14]$. The amount of virus exposure at the beginning of infection may increase the severity of the disease which is related to a higher viral load. However, as highlighted by recent studies, CT values cannot be directly affected by various factors, such as sample type, sampling time, assay design, and interpretation of the reports, and therefore must be interpreted with caution. Moreover, viral load in COVID-19 might be related to infectivity, disease phenotype, morbidity, and mortality $[15,16]$. The cycle threshold $(\mathrm{Ct})$ value obtained from a sample is an amplification measure needed for the target viral gene crossing a threshold value which can classify the viral genetic material's concentration approximately in a patient sample after rRT-PCR testing. This categorization is done as high, medium, or low. In the early phases of infection (before the capability of transmitting the infection by the patient) or late in infection when the risk of transmission is low, positive results with low viral load (high Ct) are observed. High infectivity and acute infection are presented by low Ct values (high viral load) $[15,17,18]$. Since, to date, few studies have investigated the relationships between SARS-CoV-2 viral load and mortality in a large patient cohort identification of the subpopulation with higher susceptibility to developing adverse outcomes of COVID-19, preventing the deterioration from moderate and mild to the severe conditions and reducing mortality are essential. Thus, the present study aims to investigate the demographic information, prevalence rates of common comorbidities among the negative and positive rRT-PCR patients, and the association between SARS-CoV-2 cycle threshold at hospital admission, demographic data, and outcomes of the patients in a large population in Northern Iran.

\section{Methods}

2.1. Patients and Study Design. This retrospective study was approved by the Ethics Committee of Babol University of Medical Sciences, Babol, Iran, with the ethics code IR.MUBABOL.REC.1400.012. Considering the retrospective 
nature of this study, the patients' written informed consent was waived by the committee.

This large retrospective cross-sectional study was performed between 7 March and 20 December 2020, and we collected the records of 8318 suspected COVID-19 patients who were admitted to hospitals (Ayatollah Rohani, Shahid Beheshti, Shahid Yahyanejad, and Amirkola Children Hospital) affiliated to Babol University of Medical Sciences (MUBABOL), in the north of Iran.

2.2. Data Collection. Patients' data were collected at the hospital centers using electronic medical records including demographic data, details of their medical history and comorbidities, underlying diseases, and clinical outcomes. $\mathrm{We}$ also collected the $\mathrm{Ct}$ value features for participants. Moreover, patients who did not have an oropharyngeal and nasopharyngeal swab sample, had unclear rRT-PCR results, or whose sample was analyzed on a different diagnostic platform or at a different institution were excluded.

2.3. Clinical Specimens. Oropharyngeal and nasopharyngeal samples were collected using flocked swabs from patients immediately after admission according to the World Health Organization (WHO) guidelines [19]. All samples were processed without further steps of dilution or heat inactivation according to standard laboratory biosafety guidelines. After processing, samples were divided into small volume aliquots and frozen at $-80^{\circ} \mathrm{C}$ until the time of examination.

2.4. Viral Nucleic Acid Extraction and rRT-PCR for SARSCoV-2 Detection. Laboratory confirmation of the SARSCoV-2 was made using the rRT-PCR assay. Viral RNA was extracted from $300 \mu \mathrm{L}$ of swab sample storage media using the Ribospin vRD plus Kit (GeneAll, Seoul, South Korea) according to the manufacturer's instructions. After viral RNA extraction, rRT-PCR was used to detect the presence of SARS-CoV-2 using the LightMix ${ }^{\circledR}$ SarbecoV E-gene kit (Molbiol Germany) with LightCycler Multiplex RNA Virus Master (Roche) according to the manufacturers' protocol. A cycle threshold value of $<36 \mathrm{Ct}$ was defined as a positive test result. Sensitivity is 5.2 copies per reaction. The relative viral loads of their oropharynx and nasopharyngeal swab samples were estimated with Ct based on ABI One-Step rRT-PCR results. Patients were categorized based on diagnostic $\mathrm{Ct}$ values detected from the oropharyngeal and nasopharyngeal swabs that led to a SARSCoV-2 infection diagnosis into the following three groups: Ct 10-20, Group A; Ct 21-30, Group B; and Ct 31-40, Group C.

2.5. Statistical Analysis. Categorical variables were summarized as frequencies and percentages. Continuous variables with a normal distribution were expressed as mean \pm standard deviation (SD), and chi-square and Fisher's exact tests were used to perform intergroup and categorical comparisons as appropriate. Reported $p$ values of $<0.05$ were considered statistically significant. SPSS software, version 16 (SPSS Inc., Chicago, IL, USA), was used to analyze the data.

\section{Results}

A total of 8318 suspected COVID-19 cases (48.5\% female and $51.5 \%$ male) with a mean \pm SD age of $53 \pm 25.3$ (range: 0 to 99 ) years were included in the study, of whom 4287 (51.5\%) were male and 639 (7.7\%) died of the disease during their hospital stay. Out of the 8318 suspected COVID-19 patients who were referred and hospitalized in our setting, 3250 (39.1\%) had a positive rRT-PCR result; 1632 (50.2\%) patients were male and 335 (10.3\%) patients died during their hospital stay.

The details of positive and negative rRT-PCR results based on gender $(p=0.053)$ and age $(p \leq 0.001)$ distribution and comorbidity are presented in Table 1 . Based on age distribution, most of the suspected patients were $\geq 65$ years (37.6\%), while the lowest patients belonged to the group younger than one year (2.33\%).

On the other hand, positive rRT-PCR rates increased with age, with a rate of $1 \%$ in patients aged $<1$ year, $2.6 \%$ in those aged $1-14$ years, $2.1 \%$ in those aged $15-24$ years, $20.8 \%$ in those aged 25-44 years, 38.6\% in those aged 45-64 years, and $34.9 \%$ in those aged $\geq 65$ years. Statistical analysis of age distribution showed that the occurrence of positive rRTPCR was significantly increased with age, which is shown in Table 1.

Our data revealed that $61.3 \%$ of the study's suspected patients had one or more underlying conditions such as cardiovascular disease (CVD), diabetes, and brain and neurologic disorder. Accordingly, the most frequent comorbidity reported was CVD (2818/8318; 33.9\%), followed by diabetes $(2140 / 8318 ; 25.7 \%)$ and hypertension $(1026 / 8318 ; 12.3 \%)$. On the other hand, the most prevalent comorbidities in patients with positive rRT-PCR were CVD (32\%), diabetes $(27.6 \%)$, and hypertension (13.8\%), while $35.1 \%, 24.6 \%$, and $11.4 \%$ of patients with negative rRT-PCR had CVD, diabetes, and hypertension. Statistical analysis of comorbidity distribution showed that comorbidities were significantly different in the two groups except for pregnancy $(p=0.86)$, which are shown in Table 1.

Moreover, to better demonstrate the results related to $\mathrm{Ct}$, the distribution of positive rRT-PCR revealed that 336 (10.4\%) patients had a Ct between 10 and 20 (Group A), 2464 (75.7\%) patients had a Ct between 21 and 30 (Group B), and $451(13.9 \%)$ patients had a Ct between 31 and 40 (Group C).

Ct groups differed in terms of cardiovascular disease $(p=0.06)$, hypertension $(p=0.018)$, kidney diseases $(p=0.001)$, malignancy $(p=<0.001)$, and blood disorder $(p=0.02)$; moreover, it seems that mortality was significantly different between the three groups, with the highest mortality in those with $\mathrm{Ct}$ between 10 and 20 (Group $\mathrm{A}=16.7 \%$ ) and lowest in the group with highest $\mathrm{Ct}$ (Group $\mathrm{C}=8 \%)(p<0.001)$. The $\mathrm{Ct}$ groups across age categories are shown in Table 2. Accordingly, it was noted that there was a statistically significant difference between increased age and the Ct groups. 
TABLE 1: Demographic and comorbidity information of suspected patients with COVID-19 in total population, negative and positive SARSCoV-2 rRT-PCR.

\begin{tabular}{|c|c|c|c|c|}
\hline Variable & $\begin{array}{c}\text { Total }(n=8318) \text { number } \\
(\%)\end{array}$ & $\begin{array}{c}\text { PCR negative }(n=5068) \text { number } \\
(\%)\end{array}$ & $\begin{array}{c}\text { PCR positive }(n=3250) \text { number } \\
(\%)\end{array}$ & $p$ value \\
\hline \multicolumn{5}{|c|}{ Age } \\
\hline$<1$ & $194(2.33)$ & $162(3.2)$ & $32(1)$ & \multirow{6}{*}{$<0.001$} \\
\hline $1-14$ & $542(6.5)$ & $458(9.1)$ & $84(2.6)$ & \\
\hline $15-24$ & $264(3.2)$ & $194(3.8)$ & $70(2.1)$ & \\
\hline $25-44$ & $1447(17.4)$ & $771(15.2)$ & $676(20.8)$ & \\
\hline $45-64$ & $2740(33)$ & $1489(29.4)$ & $1251(38.6)$ & \\
\hline$\geq 65$ & $3123(37.6)$ & $1990(39.3)$ & $1133(34.9)$ & \\
\hline \multicolumn{5}{|c|}{ Sex } \\
\hline Male & $4287(51.5)$ & $2655(52.4)$ & $1632(50.2)$ & \multirow{2}{*}{0.053} \\
\hline Female & $4031(48.5)$ & $2413(47.6)$ & $1618(49.8)$ & \\
\hline \multicolumn{5}{|c|}{ Outcome } \\
\hline Discharge & $7679(92.3)$ & $4764(94)$ & $2915(89.7)$ & \multirow{2}{*}{$<0.001$} \\
\hline Death & $639(7.7)$ & $304(6)$ & $335(10.3)$ & \\
\hline \multicolumn{5}{|c|}{ Comorbidity } \\
\hline Cardiovascular disease & $2818(33.9)$ & $1779(35.1)$ & $1039(32)$ & 0.003 \\
\hline Diabetes & $2140(25.7)$ & $1244(24.6)$ & $896(27.6)$ & 0.002 \\
\hline Hypertension & $1026(12.3)$ & $579(11.4)$ & $447(13.8)$ & 0.002 \\
\hline $\begin{array}{l}\text { Brain and neurologic } \\
\text { disorder }\end{array}$ & $576(6.9)$ & $434(8.6)$ & $142(4.4)$ & $<0.001$ \\
\hline Kidney diseases & $516(6.2)$ & $384(7.6)$ & $132(4.1)$ & $<0.001$ \\
\hline Malignancy & $515(6.2)$ & $416(8.2)$ & $99(3.1)$ & $<0.001$ \\
\hline Respiratory disorder & $356(4.3)$ & $251(4.6)$ & $105(3.2)$ & $<0.001$ \\
\hline GI diseases & $168(2)$ & $129(2.6)$ & $39(1.2)$ & $<0.001$ \\
\hline Blood disorder & $125(1.5)$ & $99(2)$ & $26(0.8)$ & $<0.001$ \\
\hline Liver disease & $90(1.1)$ & $65(1.3)$ & $25(0.8)$ & 0.027 \\
\hline Pregnancy & $80(1)$ & $48(1)$ & $32(1)$ & 0.86 \\
\hline Others & $173(2.1)$ & $95(1.9)$ & $78(2.4)$ & 0.1 \\
\hline No comorbidity & $3216(38.7)$ & $1805(35.6)$ & $1411(43.4)$ & \multirow{2}{*}{$<0.001$} \\
\hline$\geq 1$ comorbidity & $5102(61.3)$ & $3263(64.4)$ & $1839(56.6)$ & \\
\hline
\end{tabular}

$P$ values of $<0.05$ were considered statistically significant, and it is shown in bold.

TABLE 2: Demographic and comorbidity information of patients with positive SARS-CoV-2 rRT-PCR based on cycle threshold value (Ct).

\begin{tabular}{|c|c|c|c|c|}
\hline \multirow{2}{*}{ Variable } & \multicolumn{3}{|c|}{ Number $(\%)$ of cycle threshold value $(\mathrm{Ct})$} & \multirow{2}{*}{$p$ value } \\
\hline & $\mathrm{A}(n=336)$ & $\mathrm{B}(n=2464)$ & $\mathrm{C}(n=451)$ & \\
\hline \multicolumn{5}{|c|}{ Age } \\
\hline$<1$ & $2(0.6)$ & $15(0.6)$ & $15(3.3)$ & \multirow{6}{*}{$<0.001$} \\
\hline $1-14$ & $14(4.2)$ & $41(1.7)$ & $29(6.4)$ & \\
\hline $15-24$ & $10(3)$ & $48(2)$ & $12(2.7)$ & \\
\hline $25-44$ & $67(19.9)$ & $535(21.8)$ & $74(16.4)$ & \\
\hline $45-64$ & $110(32.7)$ & $998(40.6)$ & $144(31.9)$ & \\
\hline$\geq 65$ & $133(39.6)$ & $823(33.4)$ & $177(39.2)$ & \\
\hline \multicolumn{5}{|c|}{ Sex } \\
\hline Male & $159(47.3)$ & $1250(50.7)$ & $224(49.7)$ & \multirow{2}{*}{0.48} \\
\hline Female & $177(52.7)$ & $1214(49.3)$ & $227(50.3)$ & \\
\hline \multicolumn{5}{|c|}{ Outcome } \\
\hline Discharge & $280(83.3)$ & $2221(90.1)$ & $415(92)$ & \multirow{2}{*}{$<0.001$} \\
\hline Death & $56(16.7)$ & $243(9.9)$ & $36(8)$ & \\
\hline \multicolumn{5}{|c|}{ Comorbidity } \\
\hline Cardiovascular disease & $125(37)$ & $765(31.1)$ & $149(33)$ & 0.06 \\
\hline Diabetes & $110(32.7)$ & $664(27)$ & $122(27)$ & 0.08 \\
\hline Hypertension & $63(18.8)$ & $322(13.1)$ & $62(13.8)$ & 0.018 \\
\hline Brain and neurologic disorder & $16(4.8)$ & $104(4.2)$ & $22(4.9)$ & 0.7 \\
\hline Kidney diseases & $24(7.1)$ & $83(3.4)$ & $25(5.5)$ & 0.001 \\
\hline Malignancy & $12(3.6)$ & $60(2.4)$ & $27(6)$ & $<0.001$ \\
\hline Respiratory disorder & $9(2.7)$ & $81(3.3)$ & $15(3.3)$ & 0.83 \\
\hline GI diseases & $3(0.9)$ & $29(1.2)$ & $7(1.6)$ & 0.68 \\
\hline Blood disorder & $4(1.2)$ & $14(0.6)$ & $8(1.8)$ & 0.02 \\
\hline
\end{tabular}


TABle 2: Continued.

\begin{tabular}{|c|c|c|c|c|}
\hline \multirow{2}{*}{ Variable } & \multicolumn{3}{|c|}{ Number $(\%)$ of cycle threshold value $(\mathrm{Ct})$} & \multirow{2}{*}{$p$ value } \\
\hline & $\mathrm{A}(n=336)$ & $\mathrm{B}(n=2464)$ & $\mathrm{C}(n=451)$ & \\
\hline Liver disease & $2(0.6)$ & $19(0.8)$ & $4(0.9)$ & 0.89 \\
\hline Pregnancy & $3(0.9)$ & $23(0.9)$ & $6(1.3)$ & 0.7 \\
\hline Others & $8(2.4)$ & $61(2.5)$ & $9(2)$ & 0.8 \\
\hline No comorbidity & $126(8.9)$ & $1103(78.1)$ & $183(13)$ & \\
\hline$\geq 1$ comorbidity & $210(11.4)$ & $1361(74)$ & $268(14.6)$ & 0.01 \\
\hline
\end{tabular}

$P$ values of $<0.05$ were considered statistically significant, and it is shown in bold. A: Ct 10-20; B: Ct 21-30; C: Ct 31-40.

There is a significant difference between the outcomes of patients across different age groups. Compared with discharged alive patients, death cases showed a significantly higher prevalence of comorbidities including kidney diseases $(6.3 \%$ vs. $3.8 \%, p=0.031)$, diabetes $(34.9 \%$ vs. $26.7 \%$, $p=0.001)$, and respiratory disorder $(5.7 \%$ vs. $3 \%$, $p=0.008)$. The details of demographic information and comorbidities between discharged alive patients and death patients are shown in Table 3.

To evaluate the impact of gender in COVID-19, we compared the comorbidity and outcomes between male and female patients (Table 4). Results showed that the percentage of CVD (34.2\% vs. $29.7 \%, p=0.006)$, diabetes ( $32 \%$ vs. $23.2 \%, p=<0.001)$, and hypertension ( $16 \%$ vs. $11.5 \%$, $p=<0.001)$ was significantly higher in female patients. Moreover, the mortality in male patients was significantly more $(12.2 \%$ vs. $8.4 \%$, in males and females, respectively, $p=<0.001)$. Besides, $60.5 \%$ of the male patients had one or more comorbidities compared to female patients $(60.5 \%$ vs. $52.7 \%, p=<0.001)$. The details of demographic information and comorbidities among female and male patients are shown in Table 4.

\section{Discussion}

In this cross-sectional study, we report the demographic, clinical, and outcome characteristics of 8,318 suspected COVID-19 patients who were admitted to the hospitals affiliated to Babol University of Medical Sciences (Babol, north of Iran) during the SARS-CoV-2 pandemic. Among collected samples, real-time reverse transcriptase PCR results showed that $39.1 \%(n: 3,250 / 8,318)$ were positive for SARS-CoV-2 genome, including 50.2\% ( $n: 1,632 / 3,250)$ males and $49.8 \%$ ( $n: 1,618 / 3,250)$ females. There were no significant differences in positive rRT-PCR results between males and females $(p=0.053)$. These data are in agreement with Goshayeshi et al. (2021) [20], Allameh et al. (2020) [21], and Trunfio et al. (2021) [22].

As shown in Table 1 , the number of positive cases significantly increases with age $(p<0.001)$. Compared with the patients suspected of COVID-19, the discharge and death rates in patients with a definitive diagnosis of COVID-19 infection were $89.7 \%(n ; 2,915 / 3,250)$ and $10.3 \%(n ; 335 /$ $3,250)$, respectively, which is significant $(p<0.001)$. These results are consistent with Han et al. (2020) [23] and Bhaskaran et al. (2021) [24] who indicated that COVID-19 largely multiplies present risks faced by the patients, with some notable exceptions. Spanish researchers showed that aging is a potential risk factor for COVID-19 death. On the other hand, Azarkar et al. (2020) showed that the number of patients that are older than 60 is also positively associated with the death rate [25].Aging is independently associated with increased COVID-19 mortality, since our cases were mainly elderly with at least one comorbidity, including hypertension, CVD, diabetes, kidney diseases, respiratory disorder, malignancies, and cerebral, neurological, hepatogastrointestinal, and hematologic disorders. In our study, except for pregnancy, other comorbidities were significantly associated with death in the patients with COVID-19 $(p<0.05)$. In this regard, Mohammad Ebrahimi et al. reported malignancy and nervous and respiratory diseases to be significantly associated with increased case fatality rate. Moreover, in partial agreement with our results, their finding showed that diabetes, CVD, chronic renal diseases, nervous disease, and malignancy had higher rates in the nonsurviving group as compared with the surviving one [26].

Furthermore, in 2020, Davies et al. showed that the susceptibility to COVID-19 infection in individuals under 20 years of age is almost half that of adults over 20 years, and clinical symptoms have increased from $21 \%$ in people aged 10 to 19 years to $69 \%$ in people aged over 70 years [27]. In line with our data, Williamson et al. (2020) showed that the general cumulative prevalence of COVID-19 mortality rate 3 months after the beginning of the study was less than $0.01 \%$ in 18 - to 39 -year-olds, which increased to $0.67 \%$ and $0.44 \%$ in men and women over 80 years of age, respectively [28]. As you can see in Table 3 and in concordance with Davies et al. (2020), the death rate in SARS-CoV-2 infection is age-dependent [27]. In a study conducted in the UK, Davies et al. showed that age dependence in susceptibility to infection and the likelihood of having a clinically symptomatic exhibition of COVID-19 increased from $~ 20 \%$ in children to $\sim 70 \%$ in the elderly. Therefore, there is evidence to show that there is both age-varying susceptibility to SARS-CoV-2 infection and age-varying severity in COVID-19 cases [27].

In concordance with our findings, Williamson et al. showed that the COVID-19-related deaths with a hazard ratio of 1.59 (95\% CI 1.53-1.65) were associated with being male, being elderly, diabetes, severe asthma, and other medical complications [28]. Moreover, in case series from China, Europe, and the USA, COVID-19 hospitalizations, admission to ICU, invasive mechanical ventilation (IMV), and in-hospital deaths have consistently been higher in men than in women [29-34]. The reasons for gender differences in the consequences of COVID-19 can be as follows: (i) 
TABLE 3: Demographic and comorbidity information of patients with positive SARS-CoV-2 rRT-PCR based on discharge and death.

\begin{tabular}{|c|c|c|c|c|}
\hline Variable & Total $\left(N=3246^{*}\right)$ number $(\%)$ & $\begin{array}{c}\text { Discharged }(N=2911) \text { number } \\
(\%)\end{array}$ & Death $(N=335)$ number $(\%)$ & $p$ value \\
\hline \multicolumn{5}{|c|}{ Age } \\
\hline$<1$ & $32(1)$ & $31(1.06)$ & $1(0.3)$ & \multirow{6}{*}{$<0.001$} \\
\hline $1-14$ & $84(2.6)$ & $84(2.9)$ & $0(0.0)$ & \\
\hline $15-24$ & $70(2.1)$ & $69(2.4)$ & $1(0.3)$ & \\
\hline $25-44$ & $676(20.8)$ & $648(22.3)$ & $28(8.4)$ & \\
\hline $45-64$ & $1251(38.6)$ & $1142(39.2)$ & $109(32.5)$ & \\
\hline$\geq 65$ & $1133(34.9)$ & $937(32.2)$ & $196(58.5)$ & \\
\hline \multicolumn{5}{|c|}{ Sex } \\
\hline Male & $1632(50.2)$ & $1433(49.2)$ & $199(59.4)$ & \multirow{2}{*}{$<0.001$} \\
\hline Female & $1618(49.8)$ & $1482(50.8)$ & $136(40.6)$ & \\
\hline \multicolumn{5}{|c|}{ Comorbidity } \\
\hline Cardiovascular disease & $1039(32)$ & $919(31.5)$ & $120(35.8)$ & 0.1 \\
\hline Kidney diseases & $132(4.1)$ & $111(3.8)$ & $21(6.3)$ & 0.031 \\
\hline Diabetes & $896(27.6)$ & $779(26.7)$ & $117(34.9)$ & 0.001 \\
\hline Hypertension & $447(13.8)$ & $408(14)$ & $39(11.6)$ & 0.23 \\
\hline Malignancy & $99(3)$ & $86(3)$ & $13(3.9)$ & 0.35 \\
\hline Respiratory disorder & $105(3.2)$ & $86(3)$ & $19(5.7)$ & 0.008 \\
\hline Liver disease & $25(0.8)$ & $21(0.7)$ & $4(1.2)$ & 0.35 \\
\hline GI diseases & $39(1.2)$ & $35(1.2)$ & $4(1.2)$ & 0.9 \\
\hline Blood disorder & $26(0.8)$ & $25(0.9)$ & $1(0.3)$ & 0.27 \\
\hline Brain and neurologic disorder & $142(4.4)$ & $125(4.3)$ & $17(5.1)$ & 0.5 \\
\hline Pregnancy & $32(1.1)$ & $31(1.06)$ & $1(0.3)$ & 0.17 \\
\hline Others & $78(2.4)$ & $71(2.5)$ & $7(2.1)$ & 0.6 \\
\hline No comorbidity & $1411(43.4)$ & $1298(44.5)$ & $113(33.7)$ & $<0.001$ \\
\hline Comorbidity & $1839(56.6)$ & $1617(55.5)$ & $222(66.3)$ & \\
\hline
\end{tabular}

$P$ values of $<0.05$ were considered statistically significant, and it is shown in bold.

TABLE 4: Demographic and comorbidity information of patients with positive SARS-CoV-2 rRT-PCR based on sex.

\begin{tabular}{|c|c|c|c|c|}
\hline Variable & Total $(N=3246)^{*}$ number $(\%)$ & Female $(N=1615)$ number $(\%)$ & Male $(N=1631)$ number $(\%)$ & $p$ value \\
\hline \multicolumn{5}{|c|}{ Age } \\
\hline$<1$ & $32(1)$ & $12(0.7)$ & $20(1.2)$ & \multirow{6}{*}{0.008} \\
\hline $1-14$ & $84(2.6)$ & $36(2.2)$ & $48(2.9)$ & \\
\hline $15-24$ & $70(2.1)$ & $40(2.5)$ & $30(1.8)$ & \\
\hline $25-44$ & $676(20.8)$ & $339(21)$ & $337(20.6)$ & \\
\hline $45-64$ & $1251(38.6)$ & $663(41)$ & $588(36)$ & \\
\hline$\geq 65$ & $1133(34.9)$ & $525(32.5)$ & $608(37.3)$ & \\
\hline \multicolumn{5}{|c|}{ Outcome } \\
\hline Discharge & $2915(89.7)$ & $1482(91.6)$ & $1433(87.8)$ & \multirow{2}{*}{$<0.001$} \\
\hline Death & $335(10.3)$ & $136(8.4)$ & $199(12.2)$ & \\
\hline \multicolumn{5}{|c|}{ Variable } \\
\hline Cardiovascular disease & $1039(32)$ & $554(34.2)$ & $485(29.7)$ & 0.006 \\
\hline Diabetes & $896(27.6)$ & $518(32)$ & $378(23.2)$ & $<0.001$ \\
\hline Hypertension & $447(13.8)$ & $259(16)$ & $188(11.5)$ & $<0.001$ \\
\hline Brain and neurologic disorder & $142(4.4)$ & $74(4.6)$ & $68(4.2)$ & 0.57 \\
\hline Kidney diseases & $132(4.1)$ & $63(3.9)$ & $69(4.2)$ & 0.62 \\
\hline Respiratory disorder & $105(3.2)$ & $52(3.2)$ & $53(3.2)$ & 0.95 \\
\hline Malignancy & $99(3)$ & $53(3.3)$ & $46(2.8)$ & 0.44 \\
\hline GI diseases & $39(1.2)$ & $23(1.4)$ & $16(1)$ & 0.24 \\
\hline Blood disorder & $26(0.8)$ & $13(0.8)$ & $13(0.8)$ & 0.98 \\
\hline Liver disease & $25(0.8)$ & $15(0.9)$ & $10(0.6)$ & 0.3 \\
\hline Others & $78(2.4)$ & $50(3.1)$ & $28(1.7)$ & $<0.001$ \\
\hline Comorbidity & $1839(56.6)$ & $979(60.5)$ & $860(52.7)$ & $<0.001$ \\
\hline No comorbidity & $1411(43.4)$ & $639(39.5)$ & $772(47.3)$ & \\
\hline
\end{tabular}

*Age of 4 cases, unknown. $P$ values of $<0.05$ were considered statistically significant, and it is shown in bold. 
lifestyle differences, such as smoking, which is more common in men than in women and increases the risk of pneumonia and secondary infections after COVID-19; (ii) compared to men, the innate and acquired immune system is stronger in women; and (iii) a female sex hormone, estrogen, plays a protective role by activating the immune response and suppressing straight SARS-CoV replication [35-37]. The distribution of patients based on Ct of rRTPCR was as follows: $10.4 \%$ with Ct 10-20 (Group A), 75.7\% with Ct 21-30 (Group B), and 13\% with Ct 31-40 (Group C).

Another interesting finding of this research was the existence of a substantial connection between $\mathrm{Ct}$ and both age and in-hospital mortality rate. In agreement with our data, Choudhuri et al. (2020) showed that the SARS-CoV-2 cycle threshold at admission was found to be an independent predictor of inpatient mortality [38]. According to the value of $\mathrm{Ct}$, the highest and lowest mortality rates were related to Groups A and C, respectively. In concordance with our data, Dres et al. (2021) showed that the Ct values of RT-PCR were used as the indicators of the RNA viral load in the samples; the lower the Ct, the higher the RNA viral load [39]. We conclude that the lower the $\mathrm{Ct}$, the higher the viral load, resulting in a more viral spread, severe illness, and death. However, Rabaan et al. (2021) stated that various factors could affect $\mathrm{Ct}$ values, including (i) preanalytic variables (such as collection technique, type of specimen, sampling time, and viral kinetics), (ii) analytic variables (for example, different kits and runs, internal control, type of RT-PCR, purity of reagents, and pipetting defects), and (iii) postanalytical variables (interpreting the reports) [40].

In a study conducted in New York, Magleby et al. (2019) showed that the hospital mortality rate was $35 \%, 18 \%$, and $6 \%$ in the patients with high viral load ( $\mathrm{Ct}<25 ; n=220)$, medium viral load (Ct 25-30; $n=216$ ), and low viral load $(\mathrm{Ct}>30 ; n=242)$, respectively $[41,42]$.

Hence, the risk of IMV was $29 \%, 21 \%$, and $15 \%$ in patients with a high, medium, and low viral load $(p<0.001)$. Zheng et al. (2020) [43] and Liu et al. (2020) [44] have described higher viral loads and longer persistence of the virus in patients with severe illness, as compared to those with mild infection. Contrary to the above studies, Shah et al. (2021) [41], Guan et al. (2020) [31], Argenziano et al. (2019) [45], and Zou et al. (2020) [46] have not found any relationship of $\mathrm{Ct}$ values with disease severity. Regarding the false-negative results in RT-PCR, Shah et al. (2021) declared that decisions about the predicting severity of the disease should be based on clinical findings such as age, comorbidities, and laboratory parameters, including the absolute lymphocyte count, C-reactive protein levels, and D-Dimer levels rather than Ct value [41]. On the other hand, He et al. (2020) concluded that to recompense the potential risk of false-negative rRT-PCR, chest CT should be used for clinically suspected cases with negative primary RT-PCR [47]. The analysis of our data showed a significant association among $\mathrm{Ct}$ values and some comorbidities such as HTN, CKD, malignancy, and hematologic disorders. One of the reasons can be in terms of the age of the patients with low CT which makes the need for medical interventions and supportive measures in the elderly, especially the patients with risk factors. In support of our data, Biswas et al. (2021), in a comprehensive analysis, showed that male patients with COVID-19 were significantly related to increased risk of mortality compared to the female patients [48]. Moreover, it was reported that the patients with age $\geq 50$ years with COVID-19 were significantly associated with increased risk of mortality as compared to the patients with age $<50$ years. Besides, Biswas et al. revealed that except for liver disease, comorbid conditions such as hypertension, diabetes, kidney disease, respiratory disease, CVD, cerebrovascular disease, and cancer were significantly higher in nonsurvivors compared to survivors [48]. The present study has encountered some limitations, including a lack of some information and variables such as obesity and other complications.

In conclusion, elderly patients with lower $\mathrm{Ct}$ and at least one comorbidity and male cases were significantly associated with the increased risk for COVID-19-related mortality. Moreover, the mortality was significantly higher in patients with DM, kidney disease, and respiratory disease. The initial quantification of SARS-CoV-2 is provided as with useful ground to have better prognostic markers in the clinical management, the treatment of disease, and resource allocation.

\section{Data Availability}

Data are available on request from the authors.

\section{Ethical Approval}

This study was approved by the Research Ethics Committee of Babol University of Medical Sciences, Babol, Iran, with number code IR.MUBABOL.REC.1400.012.

\section{Conflicts of Interest}

The authors declare that there are no conflicts of interest.

\section{Authors' Contributions}

Farzin Sadeghi, Abazar Pournajaf, and Mehrdad Halaji contributed equally to this work.

\section{Acknowledgments}

This study was financially funded by the Babol University of Medical Sciences (grant number: 724133441). The authors would like to thank the Babol University of Medical Sciences for funding this study. Also, the authors would like to thank Dr. Mohammad Ali shams for English-Language editing.

\section{References}

[1] H. Zhu, L. Wei, and P. Niu, "The novel coronavirus outbreak in Wuhan, China," Global Health Research and Policy, vol. 5, no. 1 , p. $6,2020$.

[2] K. Subbarao and S. Mahanty, "Respiratory virus infections: understanding COVID-19," Immunity, vol. 52, no. 6, pp. 905-909, 2020.

[3] Y. Han, Z. Luo, W. Zhai et al., "Comparison of the clinical manifestations between different age groups of patients with 
overseas imported COVID-19," PLoS One, vol. 15, no. 12, p. e0243347, 2020.

[4] P. Brodin, "Immune determinants of COVID-19 disease presentation and severity," Nature Medicine, vol. 27, no. 1, pp. 28-33, 2021.

[5] M. D'ascanio, M. Innammorato, and L. Pasquariello, "Age is not the only risk factor in COVID-19: the role of comorbidities and of long staying in residential care homes," BMC Geriatrics, vol. 21, p. 63, 2021.

[6] C. Wang, Z. Wang, G. Wang, J. Y.-N. Lau, K. Zhang, and W. Li, "COVID-19 in early 2021: current status and looking forward," Signal Transduction and Targeted Therapy, vol. 6, no. 1, p. 114, 2021.

[7] A. Sanyaolu, C. Okorie, and A. Marinkovic, "Comorbidity and its impact on patients with COVID-19," SN Comprehensive Clinical Medicine, vol. 2, 2020.

[8] D. Baud, X. Qi, K. Nielsen-Saines, D. Musso, L. Pomar, and G. Favre, "Real estimates of mortality following COVID-19 infection," The Lancet Infectious Diseases, vol. 20, no. 7, p. 773, 2020.

[9] F. Demeulemeester, K. de Punder, M. van Heijningen, and F. van Doesburg, "Obesity as a risk factor for severe COVID19 and complications: a review," Cells, vol. 10, no. 4, 2021.

[10] S. Khateri, H. Mohammadi, R. Khateri, and Y. Moradi, "The prevalence of underlying diseases and comorbidities in COVID-19 patients; an updated systematic review and metaanalysis," Arch Acad Emerg Med, vol. 8, p. e72, 2020.

[11] B. D. Kevadiya, J. Machhi, J. Herskovitz et al., "Diagnostics for SARS-CoV-2 infections," Nature Materials, vol. 20, no. 5, pp. 593-605, 2021.

[12] P. B. van Kasteren, B. van der Veer, S. van den Brink et al., "Comparison of seven commercial RT-PCR diagnostic kits for COVID-19," Journal of Clinical Virology, vol. 128, p. 104412, 2020.

[13] Y. Pan, D. Zhang, P. Yang, L. L. M. Poon, and Q. Wang, "Viral load of SARS-CoV-2 in clinical samples," The Lancet Infectious Diseases, vol. 20, no. 4, pp. 411-412, 2020.

[14] Y. Huang, S. Chen, Z. Yang et al., "SARS-CoV-2 viral load in clinical samples from critically ill patients," American Journal of Respiratory and Critical Care Medicine, vol. 201, no. 11, pp. 1435-1438, 2020.

[15] S. Shenoy, "SARS-CoV-2 (COVID-19), viral load and clinical outcomes; lessons learned one year into the pandemic: a systematic review," World Journal of Critical Care Medicine, vol. 10, no. 4, pp. 132-150, 2021.

[16] V. P. Shah, W. H. Farah, J. C. Hill et al., "Association between SARS-CoV-2 cycle threshold values and clinical outcomes in patients with COVID-19: a systematic review and metaanalysis," Open Forum Infectious Diseases, vol. 8, no. 9, p. ofab453, 2021.

[17] S. Baggio, A. G. L'Huillier, S. Yerly et al., "Severe acute respiratory syndrome coronavirus 2 (SARS-CoV-2) viral load in the upper respiratory tract of children and adults with early acute coronavirus disease 2019 (COVID-19)," Clinical Infectious Diseases: An Official Publication of the Infectious Diseases Society of America, vol. 73, no. 1, pp. 148-150, 2021.

[18] A. Singanayagam, M. Patel, A. Charlett et al., "Duration of infectiousness and correlation with RT-PCR cycle threshold values in cases of COVID-19, England, January to May 2020," Euro Surveillance: bulletin Europeen sur les maladies transmissibles = European communicable disease bulletin, vol. 25, 2020.

[19] W. HO. Laboratory, Testing for 2019 Novel Coronavirus (2019$n \mathrm{CoV}$ ) in Suspected Human Cases, WHO, Geneva,
Switzerland, 2020, https://www.who.int/publications-detail/ laboratory-testing-for-2019-novel-coronavirus-in-suspectedhumancases-20200117.

[20] L. Goshayeshi, M. Akbari Rad, R. Bergquist et al., "Demographic and clinical characteristics of severe Covid-19 infections: a cross-sectional study from Mashhad University of Medical Sciences, Iran," BMC Infectious Diseases, vol. 21, no. 1, p. 656, 2021.

[21] S. F. Allameh, S. Nemati, R. Ghalehtaki et al., "Clinical characteristics and outcomes of 905 COVID-19 patients admitted to imam khomeini hospital complex in the capital city of tehran, Iran," Archives of Iranian Medicine, vol. 23, no. 11, pp. 766-775, 2020.

[22] M. Trunfio, F. Venuti, F. Alladio, and B. M. Longo, "Diagnostic SARS-CoV-2 cycle threshold value predicts disease severity, survival, and six-month sequelae in COVID-19 symptomatic patients," Viruses, vol. 13, no. 2, 2021.

[23] M. S. Han, J.-H. Byun, Y. Cho, and J. H. Rim, "RT-PCR for SARS-CoV-2: quantitative versus qualitative," The Lancet Infectious Diseases, vol. 21, no. 2, p. 165, 2021.

[24] K. Bhaskaran, S. Bacon, S. J. Evans et al., "Factors associated with deaths due to COVID-19 versus other causes: population-based cohort analysis of UK primary care data and linked national death registrations within the OpenSAFELY platform," The Lancet Regional Health-Europe, vol. 6, p. 100109, 2021.

[25] G. Azarkar and F. Osmani, "Clinical characteristics and risk factors for mortality in COVID-19 inpatients in Birjand, Iran: a single-center retrospective study," European Journal of Medical Research, vol. 26, no. 1, p. 79, 2021.

[26] S. MohammadEbrahimi, A. Mohammadi, R. Bergquist et al., "Epidemiological characteristics and initial spatiotemporal visualisation of COVID-19 in a major city in the Middle East," BMC Public Health, vol. 21, no. 1, p. 1373, 2021.

[27] N. G. Davies, P. Klepac, Y. Liu et al., "Age-dependent effects in the transmission and control of COVID-19 epidemics," Nature Medicine, vol. 26, no. 8, pp. 1205-1211, 2020.

[28] E. J. Williamson, A. J. Walker, K. Bhaskaran et al., "Factors associated with COVID-19-related death using OpenSAFELY," Nature, vol. 584, no. 7821, pp. 430-436, 2020.

[29] F. Mauvais-Jarvis, "Aging, male sex, obesity, and metabolic inflammation create the perfect storm for COVID-19," $D i$ abetes, vol. 69, no. 9, pp. 1857-1863, 2020.

[30] H. Peckham, N. M. de Gruijter, C. Raine et al., "Male sex identified by global COVID-19 meta-analysis as a risk factor for death and ITU admission," Nature Communications, vol. 11, no. 1, p. 6317, 2020.

[31] W.-J. Guan, Z.-Y. Ni, Y. Hu et al., "Clinical characteristics of coronavirus disease 2019 in China," New England Journal of Medicine, vol. 382, no. 18, pp. 1708-1720, 2020.

[32] S. Richardson, J. S. Hirsch, M. Narasimhan et al., "Presenting characteristics, comorbidities, and outcomes among 5700 patients hospitalized with COVID-19 in the New York city area," JAMA, vol. 323, no. 20, pp. 2052-2059, 2020.

[33] F. Mauvais-Jarvis, N. Bairey Merz, P. J. Barnes et al., "Sex and gender: modifiers of health, disease, and medicine," The Lancet, vol. 396, no. 10250, pp. 565-582, 2020.

[34] S. L. Klein, S. Dhakal, R. L. Ursin, S. Deshpande, K. Sandberg, and F. Mauvais-Jarvis, "Biological sex impacts COVID-19 outcomes,” PLoS Pathogens, vol. 16, no. 6, p. e1008570, 2020.

[35] C. Jiang, Q. Chen, and M. Xie, "Smoking increases the risk of infectious diseases: a narrative review," Tobacco Induced Diseases, vol. 18, p. 60, 2020. 
[36] S. L. Klein and K. L. Flanagan, "Sex differences in immune responses," Nature Reviews Immunology, vol. 16, no. 10, pp. 626-638, 2016.

[37] M. S. Ghare Naz, M. Banaei, S. Dashti, and F. R. Tehrani, "An overview of sex hormones in relation to SARS-CoV-2 infection," Future Virology, vol. 16, no. 8, pp. 555-564, 2021.

[38] J. Choudhuri, J. Carter, R. Nelson et al., "SARS-CoV-2 PCR cycle threshold at hospital admission associated with patient mortality," PLoS One, vol. 15, no. 12, p. e0244777, 2020.

[39] M. Dres, S. Burrel, D. Boutolleau et al., "SARS-CoV-2 does not spread through extracorporeal membrane oxygenation or dialysis membranes," American Journal of Respiratory and Critical Care Medicine, vol. 202, no. 3, pp. 458-460, 2020.

[40] A. A. Rabaan, R. Tirupathi, and A. A. Sule, "Viral dynamics and real-time RT-PCR Ct values correlation with disease severity in COVID-19," Diagnostics, vol. 11, 2021.

[41] S. Shah, T. Singhal, N. Davar, and P. Thakkar, "No correlation between $\mathrm{Ct}$ values and severity of disease or mortality in patients with COVID 19 disease," Indian Journal of Medical Microbiology, vol. 39, no. 1, pp. 116-117, 2021.

[42] R. Magleby, L. F. Westblade, and A. Trzebucki, "Impact of SARS-CoV-2 viral load on risk of intubation and mortality among hospitalized patients with coronavirus disease 2019," Clinical Infectious Diseases, vol. 73, 2020.

[43] S. Zheng, J. Fan, F. Yu et al., "Viral load dynamics and disease severity in patients infected with SARS-CoV-2 in Zhejiang province, China, January-March 2020: retrospective cohort study," BMJ, vol. 369, p. m1443, 2020.

[44] Y. Liu, L.-M. Yan, L. Wan et al., "Viral dynamics in mild and severe cases of COVID-19," The Lancet Infectious Diseases, vol. 20, no. 6, pp. 656-657, 2020.

[45] M. G. Argenziano, S. L. Bruce, C. L. Slater et al., "Characterization and clinical course of 1000 patients with coronavirus disease 2019 in New York: retrospective case series," BMJ, vol. 369, p. m1996, 2020.

[46] L. Zou, F. Ruan, M. Huang et al., "SARS-CoV-2 viral load in upper respiratory specimens of infected patients," New England Journal of Medicine, vol. 382, no. 12, pp. 1177-1179, 2020.

[47] J.-L. He, L. Luo, Z.-D. Luo et al., "Diagnostic performance between CT and initial real-time RT-PCR for clinically suspected 2019 coronavirus disease (COVID-19) patients outside Wuhan, China," Respiratory Medicine, vol. 168, p. 105980, 2020.

[48] M. Biswas, S. Rahaman, T. K. Biswas, Z. Haque, and B. Ibrahim, "Association of sex, age, and comorbidities with mortality in COVID-19 patients: a systematic review and meta-analysis," Intervirology, vol. 64, no. 1, pp. 36-47, 2021. 